\title{
Semantic Priming of Attention Focus: Evidence for Short- and Long-Term Effects
}

\author{
Stefanie Hüttermann ${ }^{1}$, Daniel Memmert ${ }^{1}$, Otmar Bock ${ }^{2}$ \\ ${ }^{1}$ Institute of Cognitive and Team/Racket Sport Research, German Sport University Cologne, Cologne, Germany \\ ${ }^{2}$ Institute of Physiology and Anatomy, German Sport University Cologne, Cologne, Germany \\ Email: s.huettermann@dshs-koeln.de
}

Received December $1^{\text {st }}$, 2011; revised January $11^{\text {th }}, 2012$; accepted January $27^{\text {th }}, 2012$

\begin{abstract}
Research on subliminal priming documents that our brain can understand words, interpret facial expressions and decode symbols even without realizing them consciously. Thus, words presented for merely a few ms can shorten the response times to semantically related target words, if compared to words with opposite meaning (e.g., Klauer \& Musch, 2003). While most previous semantic priming studies used semantic prime-target pairs of affective valence, the present study explored for the first time semantic priming effects for prime-target pairs characterizing an attentional focus. In Experiment 1, a subliminally presented prime word was followed by an above-threshold target word such that both words denoted a broad attention focus, both denoted a narrow focus, or one word denoted a broad and the other a narrow focus. Subjects had to judge the focus of the target words, and we found their response times to be shorter when the prime-target pairs were semantically congruent rather than incongruent. In Experiment 2, a block of subliminally presented prime words, all denoting a broad or all a narrow focus of attention, was followed by a block of subliminally presented target words denoting a broad or a narrow focus in a mixed sequence. Subjects had to judge the position of each prime or target, and we found their target response times to be shorter when the target was semantically congruent rather than incongruent with the preceding prime block. We concluded that semantic priming is effective, that it works for primes denoting the attention focus, and that it persists for more than just a fraction of a second.
\end{abstract}

Keywords: Semantic Priming; Attention Focus; Subliminal Priming

\section{Introduction}

Subliminal priming refers to the fact that stimuli of which subjects are not aware can nevertheless influence their behavior (Radel, Sarrazin, \& Pelletier, 2009). This influence can manifest as facilitation of responses that are congruent with the prime, and/or as degradation of responses that are incongruent with it (Meyer \& Schvaneveldt, 1971). As an example, subjects primed with smiling faces subsequently detected a happy face faster than an angry one, and vice versa (Werheid, Alpay, Jentzsch, \& Sommer, 2005). Besides figural primes such as faces, another popular category are semantic primes, i.e., words which are presented too briefly for conscious perception, but whose meaning nevertheless influences subsequent responses (e.g., Klauer \& Musch, 2003). The semantic primes in most previous studies were words that denoted affective valence; they were found to reduce the reaction times to subsequent words of the same valence below those to words of the opposite valence (Klauer, Eder, Greenwald, \& Abrams, 2007; Naccache et al., 2005; Rossell \& Nobre, 2004). The present research expands this work to adjectives representing a broad or a narrow focus of attention; to our knowledge, there has been no attempt in the past to explore the effectiveness of semantic priming for prime-target pairs denoting subjects' attention focus.

The efficiency of semantic priming has been attributed to the existence of a lexical network (Meyer \& Schvaneveldt, 1971) which stores semantically similar words in neighboring locations (Bueno \& Frenck-Mestre, 2008): a word that is not consciously perceived can nevertheless pre-activate the pertinent region of the lexical network, and thus facilitate responses to subsequent words that activate similar network locations (e.g., Kiesel, Kunde, \& Hoffmann, 2007). In analogy to previous semantic priming studies, we reasoned that subjects who were subliminally primed with words relating to a narrow focus of attention would subsequently respond faster to target words that relate to a narrow rather than to a broad focus of attention, while the opposite would be the case for subjects who were subliminally primed with words relating to a broad focus of attention. Primes and targets are paired trial-by-trial in Experiment 1 to explore immediate effects, and block-by-block in Experiment 2 to evaluate lasting effects.

\section{Experiment 1}

\section{Method}

\section{Participants}

Thirty university students (12 females and 18 males) between the ages of 19 and 28 ( $M_{\text {age }}=23.50$ years; $S D=2.74$ years $)$ took part after signing an informed consent statement. They were right-handed, healthy, and have not participated in related research before. The study was carried out in accordance with the Helsinki Declaration of 1975.

\section{Materials and Stimuli}

Primes and targets were chosen from a set of 20 adjectives describing a narrow attentional focus, and from a set of 20 adjectives describing a broad focus of attention. The choice was 
made by common consent of two persons who were given a larger list of adjectives. The word sets are shown in Appendix A. The adjectives were comprised of small letters with an average height of $0.8 \mathrm{~cm}$. Since the longest adjective had thirteen letters, all other words were prolonged to thirteen letters by leading and trailing “+” characters.

\section{Procedure}

Participants sat at a distance of about $45 \mathrm{~cm}$ from a 15 ” PC monitor, such that the visual angle of the display was about $27^{\circ}$ vertical $\times 34^{\circ}$ horizontal. They were told that the experiment concerned the speed at which people are able to affectively categorize various stimulus words. The presented words should be evaluated as quickly as possible as either describing a narrow or a broad focus of attention.

Each trial began with a masking stimulus (dot of about $3 \mathrm{~cm}$ diameter), which appeared on the screen center for $500 \mathrm{~ms}$. Following the procedure of Bargh and Chartrand (2000), a prime word was then displayed for only $32 \mathrm{~ms}$ such that participants should merely detect a flicker on the screen. The prime was followed by another dot mask for $50 \mathrm{~ms}$. Then, the target word was presented. Participants were instructed to press a response key with one hand if the target was describing a narrow focus of attention, and another key with the other hand if the target was characterizing a broad focus of attention. The target remained on the screen until the participant responded. Prime and target were congruent on some trials (e.g. Figure 1, top), and incongruent on other trials (e.g. Figure 1, bottom).

Participants were randomly subdivided into Group N, which was primed only with adjectives representing a narrow focus, and Group B, which was primed with adjectives describing a broad focus. The primes from either list were selected by a random process, and some words were therefore presented more often than others. Target words for both groups were selected from both lists under the constraint that prime and target were always different words, and that each subject received target words from both lists in equal proportions. Since an experimental session lasted 56 trials (with 20 s rest breaks after each 14 trials), each subject received 56 prime words from one list, paired with 28 target words from the same list and 28 from the other list. One half of the subjects was instructed to use their left hand for indicating "narrow" and their right hand for indicating "broad", while the opposite was the case for the other half.

\section{Data Analysis}

We defined the response time on a given trial as the interval between target appearance and key press. The mean response time of each participant to targets representing a narrow or a broad focus, respectively, was submitted to a two-way analysis of variance ANOVA with the between-factor Group (N, B) and the within-factor Target (narrow, broad).

\section{Results and Discussion}

All participants reported retrospectively that they had only perceived a flicker between the two masks. When confronted with the fact that words had been presented between masks, they all negated having seen them.

ANOVA yielded no significant effects of Group $\left(F_{1,28}=.001\right.$, $p=.979)$ or Target $\left(F_{1,28}=1.707, p=.202\right)$, but a significant Group $\times$ Target interaction $\left(F_{1,28}=69.854, p<.001, \eta^{2}=.714\right)$ :
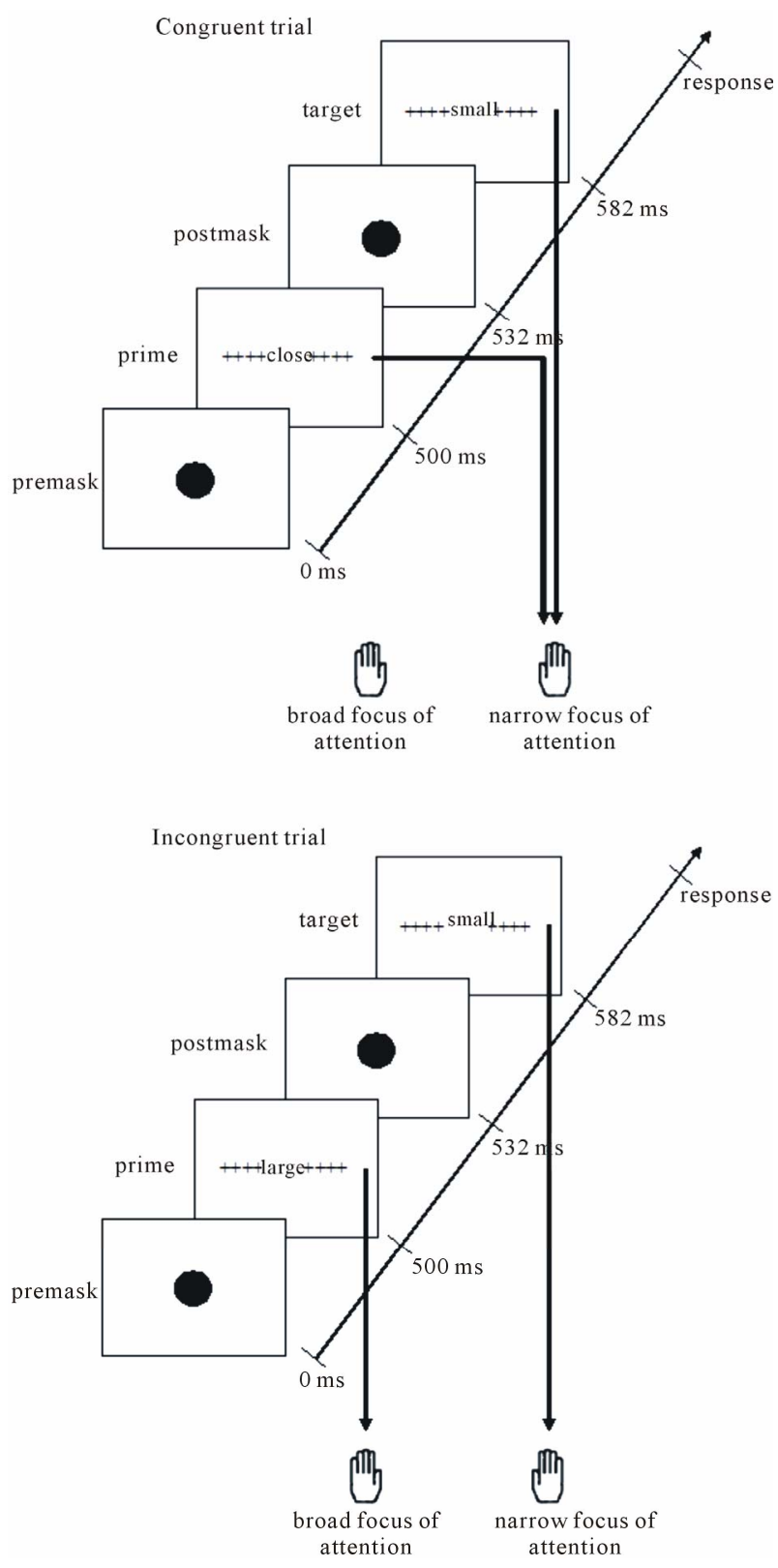

Figure 1.

Sequence of events in a congruent (top) and in an incongruent trial (bottom) in Experiment 1.

as Figure 2 illustrates, Group N responded faster to targets representing a narrow focus than to those representing a broad focus while the opposite held for Group B, i.e., each group responded faster in the primed category.

Experiment 1 confirms the effectiveness of semantic priming: responses to target words were faster when the target was preceded by a semantically congruent rather than incongruent subliminal prime word. While previous studies mostly explored the effect of affectively congruent or incongruent primes on evaluation responses to positive or negative valenced targets, the present work is, to our knowledge, the first investigating the effect of prime-target pairs denoting the attention focus. Subjects classified adjectives as representing a "broad" or a "narrow” focus, and responded faster after semantically congruent 


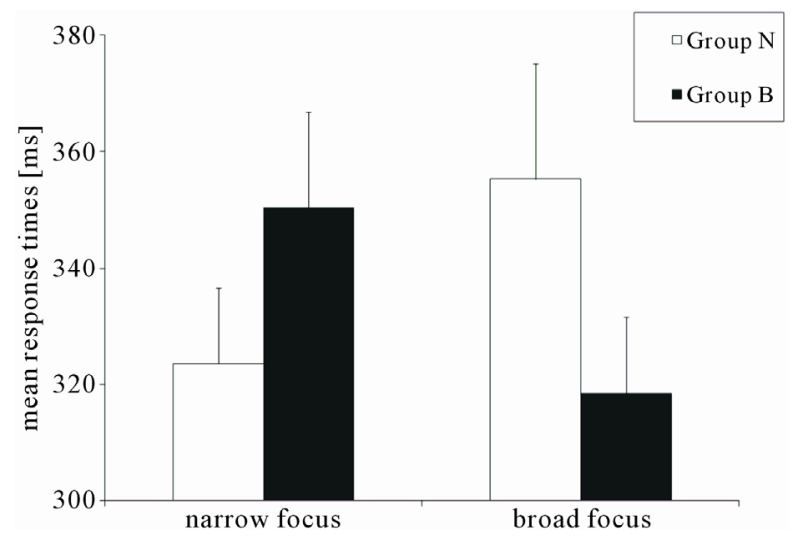

Figure 2.

Mean response times (+SD) as a function of group and above-threshold target type in Experiment 1.

rather than incongruent prime words.

\section{Experiment 2}

Experiment 1 allowed us to establish the short-term effectiveness of semantic priming, since each target was immediately preceded by a prime. Experiment 2 assessed possible long- term effects by presenting first a block of prime words referring to a given attention focus, and then a block of target words referring to either attention focus in a mixed order. To minimize a washout of priming effects during the target block, the target words were presented subliminally, and were associated with choice responses regarding their position rather than their meaning. To ensure that subjects watched all primes alertly and to harmonize experimental procedures, prime words were associated with the same choice responses as the target words. In consequence, the prime and target blocks differed only with respect to semantics (same focus versus mix of two foci), but not with respect to the required responses.

\section{Method}

\section{Participants}

Thirty-two university students (20 females and 12 males) between the ages of 20 and 29 ( $M_{\text {age }}=23.59$ years; $S D=2.59$ years) took part in the study. Written informed consent was obtained from each subject prior to participation. They were right-handed, healthy, and have not participated in related research before.

\section{Procedures}

Participants sat at a distance of about $45 \mathrm{~cm}$ from a 15” PC monitor, such that the visual angle of the display was about $27^{\circ}$ vertical $\times 34^{\circ}$ horizontal. They were told that the experiment determined the speed at which people are able to react to flashes of light.

Prime and target words were chosen from the same word sets as in Experiment 1, and were presented with the same size. In a first block, words from one given set were displayed in a random sequence, and in a second block, words from both sets in a mixed sequence. Each word was presented for $32 \mathrm{~ms}$ either 8 $\mathrm{cm}$ above or $8 \mathrm{~cm}$ below a fixation dot (of about $3 \mathrm{~cm}$ diameter) in the screen center. Due to the brevity of presentation, subjects were not aware that words were displayed and merely noticed flashes of light. Words were presented about every four seconds. Participants were instructed to release a central key and depress a key above it if they saw the flicker above the fixation dot, or depress the key below it if they saw the flicker below the fixation dot. Each block consisted of 28 words, with 20 s rest breaks between blocks. Participants were randomly subdivided into Group N', primed with adjectives representing a narrow focus, and Group B', primed with adjectives describing a broad focus of attention.

\section{Data Analysis}

The response time of a given trial was defined as the interval between word presentation and release of the central key. The mean response time to targets representing a narrow focus was calculated for each participant separately from that to targets representing a broad focus of attention. The outcome was submitted to a two-way ANOVA with the between-factor Group (N', B') and the within-factor Target (narrow, broad).

\section{Results and Discussion}

All participants reported retrospectively that they had only perceived flashes of light above or below the fixation dot, and no words. ANOVA yielded no significant effects of Group $\left(F_{1,30}=.618, p=.438\right)$ or Target $\left(F_{1,30}=.067, p=.797\right)$, but a significant Group $\times$ Target interaction $\left(F_{1,30}=14.503, p<.001\right.$, $\left.\eta^{2}=.326\right)$ : Figure 3 illustrates that Group N' primed with a narrow focus responded faster to targets with a narrow rather than to a broad focus, while the opposite was the case for Group B' primed with a broad focus.

These data show in accordance with Experiment 1 that semantic priming of target words denoting the attention focus is possible. Moreover, they document that this priming persists for more than just the fraction of a second: since the second block took about $162 \mathrm{~s}$ and priming effects were significant for the block means, we conclude that priming persisted for $81 \mathrm{~s}$ (1/2 block) or more.

\section{General Discussion}

Although a number of studies used semantic priming to induce different mental states, none of them attempted to induce a broad or narrow focus of attention, possibly because the chances of success were considered to be low. The present

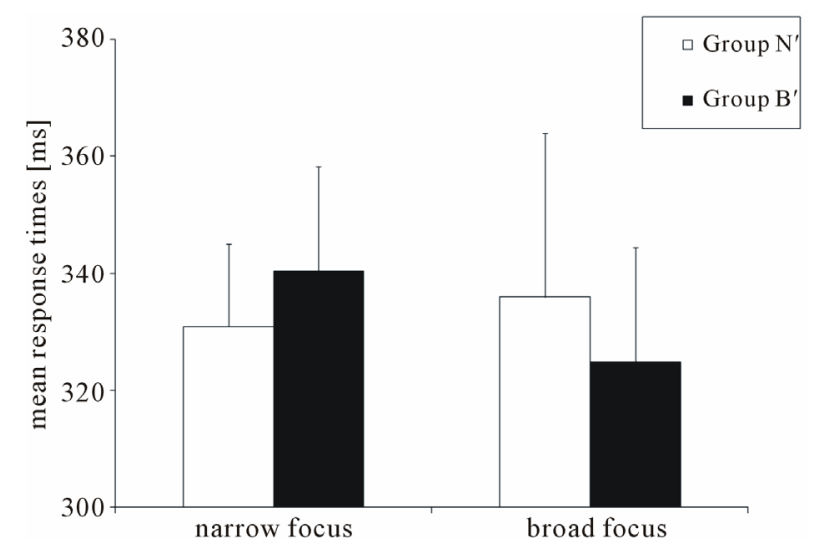

Figure 3.

Mean response times (+SD) as a function of group and subliminal target type in Experiment 2. 
research addressed this issue for the first time. In Experiment 1, participants decided whether above-threshold target words characterized a narrow or a broad focus of attention after they were primed with semantically related or unrelated words. In Experiment 2, subjects had to react as fast as possible to subliminal targets after they were primed with words denoting a narrow or broad focus. Overall, the data suggest that responses to target words of the primed category were faster than those to the non-primed category; this difference amounted to about 31 $\mathrm{ms}$ in Experiment 1, and to about $10 \mathrm{~ms}$ in Experiment 2. Two explanations for the greater effect in Experiment 1 are conceivable. First, primes were presented above threshold rather than subliminally, and thus might have activated the lexical network (see Introduction) more efficiently. Second, primes were presented in each trial rather than in separate blocks, and their effects therefore did not decay with the passage of time.

It should be noted that the present study used words to prime the attention focus, and used again words to confirm the success of priming. It would be desirable in subsequent work to validate priming effects in other ways, e.g., using visuo-spatial or manual-skill tasks, in order to find out whether the effects of priming extend beyond the semantic domain. If so, it might be interesting to explore the utility of semantic priming in everyday scenarios such as car driving. Dense traffic requires oftentimes a broad focus of attention to deal with many parallel activities on the road, but sometimes it requires a narrow focus to process a challenging event — such as an animal on the road-in great detail (Nideffer, 1976). An intelligent driver assistance system could identify potential hazards ahead, and apply semantic priming to direct the driver's attention accordingly. This might be more effective than an unspecific warning sound which alerts the drivers but doesn't modify their mental processing characteristics.

Another exemplary area of application is marketing. Several studies (e.g., Karremans, Stroebe, \& Claus, 2006; Klink, 2009) found that subliminal advertising influences consumer choice. Semantic priming of attention could be used to induce a broad focus such that consumers place a higher weight on the overall characteristics of a product, or a narrow focus such that they mainly consider product details.

\section{REFERENCES}

Bargh, J. D., \& Chartrand, T. (2000). The mind in the middle: A practical guide to priming and automaticity research. In H. T. Reis, \& C. M. Judd (Eds.), Handbook of research methods in social and per-

\section{Appendix A}

\section{Word Set 1}

nahe [close], genau [strict], akkurat [accurate], speziell [special], einzeln [sole], subtil [subtle], komprimiert [compressed], gründlich [thorough], intensiv [intense], umfriedet [enclosed], gradlinig [linear], direkt [direct], klein [small], exakt [exact], detailliert [detailed], schmal [slim], präzise [precise], gezielt [specific], kurz [short], beschränkt [limited]. sonality psychology (pp. 253-285). Cambridge: Cambridge University Press.

Bargh, J. A., Chen, M., \& Burrows, L. (1996). Automaticity of social behavior: Direct effects of trait construct and stereotype activation on action. Journal of Personality and Social Psychology, 71, 230-244. doi:10.1037/0022-3514.71.2.230

Bueno, S., \& Frenck-Mestre, C. (2008). The activation of semantic memory: Effects of prime exposure, prime-target relationship and task demands. Memory \& Cognition, 36, 882-898. doi:10.3758/MC.36.4.882

Karremans, J. C., Stroebe, W., \& Claus, J. (2006). Beyond Vicary’s fantasies: The impact of subliminal priming and brand choice. Journal of Experimental Social Psychology, 42, 792-798. doi:10.1016/j.jesp.2005.12.002

Kiesel, A., Kunde, W., \& Hoffmann, J. (2007). Mechanisms of subliminal response priming. Advances in Cognitive Psychology, 3, 307-315. doi:10.2478/v10053-008-0032-1

Klauer, K. C., Eder, A. B., Greenwald, A. G., \& Abrams, R. L. (2007). Priming of semantic classifications by novel subliminal prime words. Consciousness and Cognition, 16, 63-83. doi:10.1016/j.concog.2005.12.002

Klauer, K. C., \& Musch, J. (2003). Affective priming: Findings and theories. In J. Musch, \& K. C. Klauer (Eds.), The psychology of evaluation: Affective processes in cognition and emotion (pp. 7-50). Mahwah, NJ: Lawrence Erlbaum.

Klink, R. R. (2009). Gender differences in new brand name response. Marketing Letters, 20, 313-326. doi:10.1007/s11002-008-9066-X

Meyer, D. E., \& Schvaneveldt, R. W. (1971). Facilitation in recognizing pairs of words: Evidence of a dependence between retrieval operations. Journal of Experimental Psychology, 90, 227-234. doi:10.1037/h0031564

Naccache, L., Gaillard, R., Adam, C., Hasboun, D., Clémenceau, S., Baulac, M., Dehaene, S., \& Cohen, L. (2005). A direct intracranial record of emotions evoked by subliminal words. Proceedings of the National Academy of Sciences, 102, 7713-7717. doi:10.1073/pnas.0500542102

Nideffer, R. M. (1976). Test of attentional and interpersonal style. Journal of Personality and Social Psychology, 34, 394-404. doi:10.1037/0022-3514.34.3.394

Radel, R., Sarrazin, P., \& Pelletier, L. G. (2009). Evidence of subliminally primed motivational orientations: The effects of unconscious motivational processes on the performance of a new motor task. Journal of Sport and Exercise Psychology, 31, 651-674.

Rossell, S. L., \& Nobre, A. C. (2004). Semantic priming of different affective categories. Emotion, 4, 354-363. doi:10.1037/1528-3542.4.4.354

Werheid, K., Alpay, G., Jentzsch, I., \& Sommer, W. (2005). Priming the processing of facial affect: Event-related potentials reveal early detection of emotional expression. International Journal of Psychophysiology, 55, 209-219. doi:10.1016/j.ijpsycho.2004.07.006

\section{Word Set 2}

weit [far], ungefähr [approximate], global [global], allgemein [universal], vielfach [multiple], ausgedehnt [broad], offen [open], generell [general], üblich [common], geräumig [spacious], entfernt [distant], lang [long], groß [big], pauschal [blanket], umfassend [comprehensive], weltweit [all-round], verteilt [distributed], umfangreich [large], grob [rough], total [total]. 